Trauma Berufskrankh 2008 · 10 [Suppl 3]:352-356

DOI 10.1007/s10039-008-1415-8

Online publiziert: 27. Juni 2008

(c) Springer Medizin Verlag 2008

J. von Recum · A. Wentzensen

Klinik für Unfall- und Wiederherstellungschirurgie, BG-Unfallklinik, Unfallchirurgische Klinik an der Universität Heidelberg, Ludwigshafen

\title{
Arthroskopie ohne vorherige MRT
}

Arzt. In Zeiten knapper werdender Erlöse sollte aus Sicht des behandelnden Arztes bereits am Anfang der Therapie die Prozedur bekannt sein, die den höchsten Ressourcenverbrauch beinhaltet. Hier liegt der Schwerpunkt des Interesses auf einer möglichst vollständigen Auslastung des OP und einer Therapie in einer Sitzung. Hierfür ist ein möglichst exakter Therapieplan erforderlich.

\section{Diagnostik} gien an Bedeutung gewonnen. Ihre hohe Akzeptanz ist insbesondere in der Tatsache begründet, dass sie nicht mit einer Strahlenbelastung für den Patienten verbunden ist. Mit ihrer zunehmenden Verfügbarkeit entstehen aber zusätzliche Behandlungskosten, die im Rahmen der knappen Ressourcen kritisch hinterfragt werden sollten. Insbesondere muss geklärt werden, ob in Fällen eines negativen Untersuchungsergebnisses auf eine Arthroskopie zur weiteren Abklärung verzichtet werden kann. Dabei sind zunächst die unterschiedlichen Interessenlagen der involvierten Parteien von Bedeutung:

Patient. Sein Hauptinteresse ist sicher in einer Restitutio ad integrum zu sehen. Dabei sollen unnötige Eingriffe vermieden werden, ohne jedoch das Risiko einzugehen, Verletzungen und Erkrankungen nicht zu erkennen.

Kostenträger. Aus seiner Sicht gilt es, unnötige Kosten zu vermeiden. Im Fall der gesetzlichen Unfallversicherung kommt der Wunsch nach dem frühestmöglichen Wiedereintritt der Arbeitsfähigkeit hinzu. Hier gilt es, Verzögerungen durch unnötige Untersuchungen zu verhindern.
Bei Erkrankungen und Verletzungen des Kniegelenks stehen für die Diagnostik zur Verfügung:

- Anamneseerhebung,

- klinische Untersuchung,

- bildgebende Verfahren und

- diagnostische Endoskopien.

\section{Klinische Untersuchung}

In Kombination mit der Anamneseerhebung können eine Vielzahl von Veränderungen erkannt werden. Der Vorteil der klinischen Untersuchung ist sicher in ihrer leichten Anwendbarkeit, ihrer flächendeckenden Verfügbarkeit und ihrer geringen Invasivität zu sehen. Allerdings sind ihre Sensitivität und Spezifität untersucherabhängig. Ihre diagnostische Genauigkeit nimmt in der Reihenfolge Ligamente - Menisken - Knorpel ab.

\section{MRT}

In den letzten Jahren etablierte sie sich zur Methode der Wahl in der Diagnostik intraartrikulärer Gelenkpathologien, v. a. nach Verletzungen (Bandruptur) und bei Meniskusschäden. Sie steht heute unein- geschränkt und flächendeckend zu Verfügung [7].

Die MRT ist insbesondere geeignet, die weichteiligen Strukturen des Kniegelenks sowie Bänder, Menisken, Knorpel und Synovia darzustellen. Sie hat ihre Stärke darüber hinaus in der Detektion so genannter „okkulter Frakturen“ oder Spongiosafrakturen, die sich in Form der so genannten „bone bruises“ darstellen. Der Krankheitswert dieser MRT-Diagnose ist umstritten. Zur Beurteilung des Frakturausmaßes ist sie aufgrund der geringeren Ortsauflösung und der systembedingten Überzeichnung der Frakturlinien durch das frakturbedingte Ödem dem CT unterlegen. Nachteilig wirken sich die zusätzlichen Kosten und die zeitliche Verzögerung bis zur definitiven Therapie aus. Sensitivität und Spezifität hängen von der Feldstärke des Geräts sowie vom verwendeten Protokoll ab. Die diagnostische Genauigkeit ist in hohem Maß untersucherabhängig.

\section{Arthroskopie}

Sie gilt als Goldstandard in der Diagnostik und Therapie von Pathologien des Kniegelenks. Sie bietet durch die direkte Visualisierung der intraartikulären Strukturen und die Möglichkeit der Palpation der Oberflächenbeschaffenheit die verlässlichste Information. Allerdings bleibt die Beurteilung auf die Oberflächen beschränkt. Subchondral gelegene Veränderungen werden durch die Arthroskopie nicht erfasst. Ein besonderer Vorteil ist in der sofortigen therapeutischen Option dieses Verfahrens zu sehen.

Die Arthroskopie stellt jedoch eine invasive Untersuchung dar, die zu ihrer 
Durchführung einer Narkose bedarf und Komplikationsmöglichkeiten beinhaltet. Je nach Literaturstelle wird der Anteil an rein diagnostischen Arthroskopien ohne Intervention mit zwischen 39\% und 73\% beziffert. Diese Eingriffe scheinen vermeidbar und führen evtl. zu einer Invalidisierung gesunder Patienten.

Durch die Einführung und den zunehmenden Einsatz der MRT hat die diagnostische Arthroskopie bereits an Bedeutung verloren. Sie hat ihren Stellenwert aber noch bei unklaren, durch die üblichen diagnostischen Maßnahmen nicht zu klärenden Beschwerden, posttraumatisch bei Hämarthros ohne richtungweisende Diagnose und im Rahmen anderer gelenknaher Eingriffe, wie Umstellungsosteotomien oder Osteosynthesen von Gelenkfrakturen, behalten [8].

\section{Auswahl der optimalen diagnostischen Methode}

Ziel dieser Literaturrecherche war es, die diagnostische Genauigkeit der Untersuchungsmethoden bei Verletzungen des Kniegelenks am „Goldstandard Arthroskopie“ $\mathrm{zu}$ messen. Insbesondere sollte geklärt werden, ob eine nicht richtungsweisende klinische und eine unauffällige magnetresonanztomographische Untersuchung eine Arthroskopie des Kniegelenks entbehrlich machen. Eingeschlossen wurden Arbeiten, in welchen die diagnostische Genauigkeit der Untersuchungsmethoden mit der Arthroskopie verglichen worden war.

\section{Kreuzband}

Zu den häufigsten Verletzungen des Kniegelenks gehören die der Kreuzbänder. Aufgrund deren hoher Inzidenz kommt der sicheren klinischen Diagnostik ein hoher Stellenwert zu.

\section{Klinische Untersuchung}

Benjaminse et al. [2] überprüften anhand der Literatur die diagnostische Genauigkeit der klinischen Tests zur Untersuchung der Kreuzbandinstabilität. Eingeschlossen wurden 28 Studien, die mindestens einen diagnostischen Test mit einem Standardverfahren - in diesem Kontext

Trauma Berufskrankh 2008 • 10 [Suppl 3]:352-356 DOI 10.1007/s10039-008-1415-8

(c) Springer Medizin Verlag 2008

J. von Recum · A. Wentzensen

\section{Arthroskopie ohne vorherige MRT}

\section{Zusammenfassung}

Durch die flächendeckende Etablierung der Magnetresonanztomographie (MRT) haben die Arthroskopie als rein diagnostisches Verfahren sowie die klinische und radiologische Untersuchung als primäres Diagnostikum an Bedeutung verloren. Durch einen unkritischen Einsatz der MRT werden jedoch unnötige Kosten produziert, Therapien verzögert und z. T. Arbeitsunfähigkeitszeiten verlängert. Die Erkrankungen des Bandapparats sind für den geübten Untersucher durch eine strukturierte klinische Befunderhebung sicher zu erfassen, eine MRT-Diagnostik erübrigt sich bei positivem Befund und zeitnaher operativer Therapie. Bei verzögerter definitiver Behandlung sind zeitkritische Begleitverletzungen auszuschließen. Die Treffsicher-

\section{Arthroscopy without prior MRT}

\section{Abstract}

As a result of the widespread establishment of magnetic resonance tomography (MRT) in diagnostics, arthroscopy, as well as clinical and radiological examinations, have lost their significance as primary diagnostic modalities. However, due to the use of MRT in non-critical cases, unnecessary costs are produced, therapy is delayed and, in some cases, periods of disability are extended. Diseases of the palmar ligaments are straightforward for the practiced examiner to diagnose by means of structured clinical diagnostic steps; diagnosis with MRT is unnecessary in the presence of positive findings and prompt surgical therapy. In the case of delayed definitive treatment, concomitant injuries which are critical in terms of time need to be excluded. Accura- heit bei der klinischen Diagnostik von Meniskusschäden ist weniger verlässlich, dagegen erreichen Sensitivität und Spezifität der MRTDiagnostik bei geeigneten Protokollen hohe Werte. Bei negativem MRT-Befund kann eine zusätzliche Arthroskopie unterbleiben. Knorpelläsionen sind klinisch schlecht zu erfassen, v. a. sind Aussagen zur Tiefe und Ausdehnung erschwert. Die MRT weist gute Werte für die Spezifität bei schlechter Sensitivität und gleichzeitiger Tendenz zur Überbewertung des Läsionsgrades auf.

\section{Schlüsselwörter}

Bildgebende Diagnostik · Bandapparat ·

Zeitkritische Begleitverletzungen .

Meniskusschäden $\cdot$ Knorpelläsionen cy in the clinical diagnosis of meniscus injury is less dependable, while on the other hand, sensitivity and specificity in MRT, when the appropriate protocols are used, reach high values. In the case of negative MRT findings, additional arthroscopy can be dispensed with. Cartilage lesions are more difficult to assess, in particular in terms of depth and extent. MRT provides helpful values in terms of specificity when sensitivity is poor, showing at the same time a tendency to overestimate degree of lesions.

\section{Keywords}

Diagnostic imaging - Palmar ligaments . Time-critical concomitant injuries - Meniscus injury - Cartilage lesions 
Tab. 1 Genauigkeit klinischer Untersuchungsverfahren in der Meniskusdiagnostik

\begin{tabular}{lll}
\hline Test & Sensitivität [\%] & Spezifität [\%] \\
Apley-Grinding-Test & $16-41$ & $86-93$ \\
\hline $\begin{array}{l}\text { Druckschmerzen über dem Gelenkspalt } \\
\text { (,joint line tenderness") }\end{array}$ & $67-88$ & $30-97$ \\
\hline McMurray-Zeichen & $16-67$ & $86-98$ \\
\hline
\end{tabular}

Arthroskopie, Arthrotomie und MRT evaluiert hatten.

Der Lachman-Test ist nach dieser Metaanalyse [2] die verlässlichste Methode zum Nachweis einer Kreuzbandinstabilität. Die über alle Studien gepoolte Sensitivität lag bei $85 \%$ [ $95 \%$-Konfidenzintervall (95\%-CI) 83-87], und die gepoolte Spezifität betrug $94 \%(95 \%-\mathrm{CI}=92-95)$. Der Pivot-Shift-Test wies mit 98\% (95\%$\mathrm{CI}=$ 96-99) die höchste Spezifität auf, war aber mit $24 \%$ (95\%-CI=21-27) wenig sensitiv. Bei chronischen Instabilitäten erreichte der Schubladentest eine hohe Sensitivität und Spezifität [ $92 \%$ ( $95 \%$-CI=8895) und $91 \%$ (95\%-CI=87-94)], war aber bei akuten Verletzungen kaum verwertbar. Die Autoren schlussfolgerten, dass die klinische Untersuchung die Kombination von Lachman- und Pivot-Shift-Test beinhalten sollte.

Im Fall eines positiven Befundes kann aus unserer Sicht auf eine weiterführende magnetresonanztomographische Diagnostik verzichtet werden, wenn sich die Therapie in Form einer Gelenkspiegelung und Ersatzplastik innerhalb eines kurzen Zeitrahmens anschließt. Sollte die Behandlung nicht zeitnah erfolgen, sind zeitkritische Begleitverletzungen wie Knorpel- und Meniskusverletzungen durch weiterführende Diagnostik auszuschließen.

\section{MRT}

In einer Metaanalyse wurde ihre klinische Genauigkeit bezüglich der Identifikation von Verletzungen des vorderen Kreuzbandes überprüft. Eingeschlossen wurden 10 prospektive Arbeiten, in welchen der MRT-Befund arthroskopisch überprüft worden war. In den Arbeiten konnten eine Sensitivität von $100 \%$ und eine Spezifität von $96 \%$ festgestellt werden. Der positive Vorhersagewert für eine Kreuzbandverletzung wurde mit $80 \%$, der negative Vorhersagewert mit 98\% kalkuliert [2].
Der hohe "negative Vorhersagewert", der in der Mehrzahl der Studien 100\% beträgt, lässt nach unserer Einschätzung den Verzicht auf eine diagnostische Arthroskopie zu.

\section{Meniskus}

\section{Klinische Untersuchung}

Zur Beurteilung der Menisken steht eine Reihe von Tests zur Verfügung. Neben einem lokalisierten Druckschmerz über dem Gelenkspalt finden die Meniskuszeichen nach Steinmann (I und II) und nach McMurray sowie der Apley-GrindingTest Anwendung.

Ryzewicz et al. [9] schlossen in eine Metaanalyse 32 prospektive Studien ein, in welchen die klinische Untersuchung auf Meniskusläsionen mittels Arthroskopie überprüft worden war. Untersucht wurden Druckschmerzen über dem Gelenkspalt, der Apley-Grinding-Test und das McMurray-Zeichen. Die Ergebnisse waren sehr heterogen (- Tab. 1). Auffällig war die Verschlechterung der diagnostischen Genauigkeit bei akuten Verletzungen und dem gleichzeitigen Vorliegen von ligamentären Schädigungen.

\section{MRT}

Durch ihre Einführung hat die bildgebende Untersuchung der Menisken an Stellenwert gewonnen. Waren diese zuvor nur durch Anwendung von Kontrastmitteln oder Instillation von Luft in das Gelenk bildtechnisch indirekt abbildbar, können sie nun magnetresonanztomographisch direkt dargestellt werden. Ryzewicz et al. [9] werteten in ihrer Metaanalyse 32 prospektive Studien aus, in welchen magnetresonanztomographische Befunde arthroskopisch gesichert worden waren. Die Studien wurden unabhängig von der Feldstärke des verwendeten Geräts eingeschlossen, Voraussetzung war aber die
Verwendung eines geeigneten Protokolls. Die Auswertung der Studien ergab ein inhomogenes Bild. In Abhängigkeit von der Feldstärke und der Spezialisierung der auswertenden Radiologe wurden eine Sensitivität von 40-100\% und eine Spezifität von $66-100 \%$ festgestellt. Der positive Vorhersagewert betrug in den Studien 67$100 \%$, der negative $73-100 \%$.

Betrachtet man nur Publikationen, in welchen Tomographen mit 3,0 T Feldstärke verwendet und die von auf muskuloskelettale Befundung spezialisierten Radiologen ausgewertet worden waren, finden sich eine Sensitivität von $96 \%$ und eine Spezifität von $97 \%$ [6].

\section{Knorpel}

\section{Klinische Untersuchung}

Für die klinische Beurteilung des Gelenkknorpels stehen die Auslösung lokaler Druckschmerzen sowie hör- und fühlbare Reibegeräusche am Ort der Entstehung oder fortgeleitet zur Verfügung. In der Literatur finden sich kaum Publikationen, in welchen klinische und arthroskopische Untersuchungen des Gelenkknorpels verglichen wurden, eine Metaanalyse fehlt aufgrund der wenigen Literaturstellen völlig.

Ike u. O'Rourke [4] untersuchten in einer prospektiven Studie 20 klinisch auffällige Patienten hinsichtlich Knorpelschäden des Kniegelenks. Die Befunde wurden anschließend arthroskopisch verifiziert. In der Auswertung wurde zwischen Patellofemoral- und Femurotibialgelenk unterschieden. Die Auswertung der Untersuchung ergab für das Patellofemoralgelenk eine Sensitivität von $69 \%$ und eine Spezifität $59 \%$, für das Femurotibialgelenk eine Sensitivität von $67 \%$ und eine Spezifität von $40 \%$. Die Verfahren waren nicht geeignet, den Schaden zu lokalisieren [4].

Nach unserer Erfahrung stehen im Fall einer akuten, traumatischen Knorpelschädigung Schwellung, Erguss und Schmerzen im Vordergrund. Eine gezielte klinische Untersuchung zur Bestimmung des Ausmaßes und der Lokalisation der Schädigung ist kaum möglich. Lediglich der chronische Knorpelschaden ist einer gezielten, klinischen Untersuchung zugänglich. Ein positiver Untersuchungs- 
befund lässt aber keine Aussagen über die Defekttiefe und die Ausdehnung zu. Durch Überprüfung desselben in verschiedenen Beugepositionen des Kniegelenks lässt sich in vielen Fällen der geschädigte Gelenkpartner identifizieren.

Der lokalisierte Knorpelschaden ist häufig durch so genannte Tissue-Response-Verfahren und Knorpelzelltransplantationen behandelbar. Das Ausmaß der Knorpelschäden lässt sich arthroskopisch verifizieren und häufig in gleicher Sitzung therapieren. Im Fall einer positiven Untersuchung ohne Hinweis auf weitergehende Gelenkpathologien ist eine weiterführende Diagnostik im Sinne einer MRT-Diagnostik entbehrlich.

\section{MRT}

Die Beurteilung des Gelenkknorpels in der MRT wird unterschiedlich bewertet. Während Geräte mit geringer Feldstärke wenig geeignet erscheinen, diesen direkt darzustellen, wird die Beurteilung mit Tomographen ab 1,5 $\mathrm{T}$ als gut beurteilt.

Figueroa et al. [3] kontrollierten die Genauigkeit der MRT-Befunde eines 1,5T-Geräts bezüglich der Knorpelschäden arthroskopisch. Bei 82 Patienten mit 115 Knorpelläsionen konnten sie eine Sensitivität von $40 \%$ bei einer Spezifität von $100 \%$ feststellen, wobei sie eine Tendenz zur Überbewertung niedriggradiger Läsionen aufzeigten.

Kuikka et al. [5] publizierten 2006 für traumatische Knorpelläsionen eine Sensitivität von $35,6 \%$ und eine Spezifität von 91,2\% unter Verwendung eines 1,0-T-Tomographen. Sie konnten eine deutliche Verschlechterung der Vorhersagewahrscheinlichkeit bei Vorhandenseins eines Hämarthros feststellen.

\section{Einfluss des Untersuchers}

Die Aussagekraft der magnetresonanztomographischen Untersuchung wird wesentlich von der Erfahrung des Untersuchers und dem angewendeten Protokoll mitbestimmt. Die Mehrheit der zitierten Studien wurde von auf die muskulo-skelettale Befundung spezialisierten Radiologen durchgeführt.

Die Realität in der unfallchirurgischorthopädischen Praxis stellt sich jedoch anders dar: Ben-Galim et al. [1] schlossen in ihre Untersuchung 139 Patienten, die aufgrund pathologischer MRT-Befunde arthroskopiert worden waren, ein. Die MRT-Befunde waren von 18 Instituten erstellt und von 12 allgemeinen Radiologen befundet worden. Der intraoperative Abgleich ergab falsch-positive Befunde für

- den Innenmeniskus in 65\%,

- den Außenmeniskus in 43\%,

- das vordere Kreuzband in $47 \%$ und

- den Gelenkknorpel in $42 \%$.

Insgesamt wurde eine Rate von 37\% unnötigen Operationen errechnet.

\section{Diskussion}

Akute und chronische Schäden des Kniegelenks sind häufige Krankheitsbilder in der täglichen Praxis. Röntgenuntersuchungen können knöcherne Veränderungen im Sinne von Frakturen oder fortgeschrittenen degenerativen Veränderungen aufdecken.

Die klinische Untersuchung wartet mit einer Vielzahl von Tests zur Beurteilung aller Strukturen des Kniegelenks auf. Deren Präzision hängt ganz wesentlich von der Erfahrung des Untersuchers ab. Grundsätzlich ist die Beurteilung des frisch verletzten Gelenks schwieriger als die des chronisch veränderten. Für die Befundung der ligamentären Stabilität wird eine hohe Genauigkeit erzielt. Bei der Beurteilung der Kreuzbandinstabilität werden Werte für die Sensitivität von $85 \%$ und für die Spezifität von 94\% erreicht. Im Fall eines positiven Befundes kann aus unserer Sicht auf eine weiterführende magnetresonanztomographische Diagnostik verzichtet werden, wenn sich die Therapie in Form einer Gelenkspiegelung und Ersatzplastik innerhalb eines kurzen Zeitrahmens anschließt. Sollte die Behandlung nicht zeitnah erfolgen, sind zeitkritische Begleitläsionen wie Knorpel- und Meniskusverletzungen durch weiterführende Diagnostik auszuschließen. Im Fall eines Hämarthros und klinisch unauffälligen ligamentären Befundes sollte eine weitere bildgebende Diagnostik angeschlossen werden. Der positive Vorhersagewert der Magnetresonanztomographie für eine vordere Kreuzbandverlet- zung beträgt $80 \%$, der negative Vorhersagewert 98\%. Der hohe „negative Vorhersagewert", der in der Mehrzahl der Studien bei $100 \%$ liegt, lässt nach unserer Einschätzung den Verzicht auf eine diagnostische Arthroskopie zu.

Die klinische Beurteilung der Menisken und des Gelenkknorpels fällt gegenüber diesen Werten deutlich ab. In unseren Augen lässt weder ein negativer noch ein positiver Befund ausreichend Rückschlüsse auf die tatsächliche Pathologie zu. Es wurden hohe Raten sowohl für falschpositive als auch für falsch-negative Befunde berichtet. Wir empfehlen bei wenig aussagekräftigen klinischen Untersuchungsbefunden und andauernden klinischen Beschwerden die weiterführende Abklärung mittels MRT. Diese zeigt bei geeigneten Protokollen und spezialisierten Untersuchern für die Beurteilung der Menisken Werte für die Sensitivität von $96 \%$ und für die Spezifität von bis zu $97 \%$. Hier kann aufgrund der geringen Rate an falsch-negativen Befunden und der systembedingten Überzeichnung der Gelenkpathologien auf eine arthroskopische Abklärung bei negativem Befund verzichtet werden.

Laut eigener Erfahrung stößt die Magnetresonanztomographie als diagnostische Methode bei undislozierten Korbhenkelrissen des Außenmeniskus an ihre Grenzen. Hier sehen wir die Ausnahmeindikation zur Arthroskopie bei negativem MRT-Befund, aber eindeutiger Vorgeschichte.

Die diagnostische Genauigkeit der Magnetresonanztomographie erreicht für die Beurteilung von Knorpelläsionen Werte für die Sensitivität von $40 \%$ und für die Spezifität von $90-100 \%$. Insgesamt fallen eine Tendenz zur Überbewertung von intraartikulären Pathologien und eine niedrige Rate an falsch-negativen Befunden auf. Aus diesem Grund kann aus unserer Sicht in solchen Fällen von einer weiteren Abklärung durch eine diagnostische Arthroskopie abgesehen werden.

\section{Fazit für die Praxis}

Durch die Einführung und flächendeckende Etablierung der MRT hat die Arthroskopie als rein diagnostisches Verfahren an Bedeutung verloren. Der ope- 
rative Eingriff „Arthroskopie“ sollte immer erst nach Abschluss einer umfassenden Diagnostik und mit therapeutischer Intention erfolgen.

Auf eine weiterführende, über die klinische Untersuchung hinausgehende Diagnostik kann immer dann verzichtet werden, wenn durch die bisherigen Untersuchungen diejenige Prozedur bekannt ist, die den höchsten Ressourcenverbrauch bedeutet.

Grundsätzlich kann bei Patellaluxationen und klinisch eindeutigen Rupturen des zentralen Bandapparats eine MRT-Diagnostik unterbleiben. Wenn aber z. B. bei der Kreuzbandruptur eine verzögerte operative Behandlung angestrebt wird, müssen zeitkritische Begleitverletzungen wie Meniskus- oder Knorpelläsionen ausgeschlossen werden.

\section{Korrespondenzadresse}

\section{Dr. J. von Recum}

Klinik für Unfall- und Wiederherstellungschirurgie, BG-Unfallklinik, Unfallchirurgische Klinik an der Universität Heidelberg, Ludwig-Guttmann-Straße 13, 67071 Ludwigshafen recum@bgu-ludwigshafen.de

Interessenkonflikt. Der korrespondierende Autor gibt an, dass kein Interessenkonflikt besteht.

\section{Literatur}

1. Ben-Galim P, Steinberg EL, Amir H et al. (2006) Accuracy of magnetic resonance imaging of the knee and unjustified surgery. Clin Orthop Relat Res 447: 100-104

2. Benjaminse A, Gokeler A, Schans CP van der (2006) Clinical diagnosis of an anterior cruciate ligament rupture: a meta-analysis. J Orthop Sports Phys Ther 36: 267-288

3. Figueroa D, Calvo R, Vaisman A et al. (2007) Knee chondral lesions: incidence and correlation between arthroscopic and magnetic resonance findings. Arthroscopy 23: 312-315

4. Ike R, O'Rourke KS (1995) Compartment-directed physical examination of the knee can predict articular cartilage abnormalities disclosed by needle arthroscopy. Arthritis Rheum 38: 917-925

5. Kuikka PI, Kiuru MJ, Niva MH et al. (2006) Sensitivity of routine 1.0-Tesla magnetic resonance imaging versus arthroscopy as gold standard in fresh traumatic chondral lesions of the knee in young adults. Arthroscopy 220: 1033-1039

6. Magee T, Williams D (2006) 3.0-T MRI of meniscal tears. AJR Am J Roentgenol 187: 371-375

7. Potter HG, Foo LF (2006) Magnetic resonance imaging of articular cartilage: trauma, degeneration and repair. Am J Sports Med 34: 661-677
8. Recum J von, Wentzensen A (2007) Arthroskopische Operationen des Kniegelenks. OP J 23: 1621

9. Ryzewicz M, Peterson B, Siparsky PN et al. (2007) The diagnosis of meniscus tears: the role of MRI and clinical examination. Clin Orthop Relat Res 455: 123-133 